\title{
Log10 Enzyme-Linked Immunosorbent Assay Unit per Dose
}

National Cancer Institute

\section{Source}

National Cancer Institute. Log10 Enzyme-Linked Immunosorbent Assay Unit per Dose.

NCI Thesaurus. Code C68879.

A logarithmic-scale (base 10) unit for measuring potency of immunologically active substance in a product determined as reactivity in a quantitative immunoassay for particular antigen or antibody and expressed per quantity of preparation used as a single dose. 